\title{
DOUBLE TTRANSOBTURATOR APPROACH TO TREATING CYSTOCELE
}

\author{
S. Bandiera, G. Raciti, A. Aloisi, M. Arena, R. Giordano, \\ G. Giunta, M.G. Matarazzo, R. Morello, F. Rapisarda, \\ S.G. Vitale, A. Cianci \\ Department of Gynaecology and Microbiology \\ S. Bambino Hospital, Catania University, Catania, Italy
}

Key words

Pelvic organ prolapse, Transobturator route, Mesh erosion, Polypropylene, Acellular Porcine Derma

\section{SUMMARY}

The aim of this study is to show the safety and efficacy of the double transobturator approach, a new technique for anterior vaginal wall prolapse, using several different kinds of meshes (synthetic and biological). This is a retrospective study of 74 women treated between 2005 and 2007. The patients underwent a 3, 6 and 12 month follow-up. The anatomical cure rate, defined as grade of prolapse $<2$, was $79.7 \%$. Fifteen patients had a recurrence, 12 were treated with biological VS meshes and 3 were treated with synthetic meshes. Vaginal erosion was reported in $5(6.7 \%)$ of these patients, two of whom were treated with topic estrogenic therapy, and three of whom with partial excision. These results suggest that this technique is safe and efficacious and that the best meshes to use are synthetic meshes, because they have a lower recurrence rate than biological meshes. 


\section{INTRODUCTION}

According to the most recent epidemiological data urogenital prolapse affects about $45 \%$ of women in post menopause [1]. This incidence is increasing more and more, due to the extension of average life, hence the use of surgical therapy too. The risk of surgery for prolapse has been estimated to be around $11.1 \%$ in a woman's life [2]. During the last years, new surgical techniques have been developed, and the use of prostheses has been introduced in urogynaecological surgery. Access for positioning the meshes has changed from the classical abdominal approach to the simpler vaginal one, related to lower morbidity, a reduction of postoperative pain and a shorter period of hospitalisation[3]. The introduction of a prosthesis was a result of the high recurrence rate of anterior colporrhaphy, which is around 40\% [4]. Therefore suspension to the sacrospinous ligament may cause lesions of the pudendal vessel and sacral nerve.

Prostheses can be divided in two groups: synthetic non absorbable meshes and biological absorbable meshes.

The main characteristics of the prostheses are:

- pore size: this determines the resistance of the mesh to infection and its flexibility. If pore size is less than $10 \mu \mathrm{m}$, the meshes are called "microporous", if it is greater than $75 \mu \mathrm{m}$ they are called "macroporous". These latter meshes are best because they allow the migration of macrophages and leukocytes within them, thus reducing the risk of infection, The cause of the most common complication related to the use of synthetic materials is erosion

- density (stiffness): this depends on the pore size: microporous and multifilament meshes have an elevated density that increases the probability of erosion. Density is the opposite of elasticity. The denser the mesh, the less flexible it is, so it will be less integrated into the host tissue, increasing the probability of erosion. On the other hand low density meshes will be more integrated in the host tissue, with a lower rate of erosion.

- composition: monofilament or multifilament

- interstice: this represents the distance between the single filaments of the material; if it is less than 10 $\mu \mathrm{m}$ the material is considered a microporous mesh.

Although these characteristics are important for determining the incidence of erosion, the main aspect to be considered is the interaction with the tissue interface. The ideal prosthesis should induce a minimal initial inflammatory reaction followed by vascular and fibroblastic infiltration. Otherwise the phenomenon of encapsulation occurs, which reduces its effectiveness and increases the risk of erosion.

Biological materials can be divided into:

- autologous, (rectus fascia, fascia lata, and vaginal skin)

- allografts, (dura mater and dermis) 
- xenografts, porcine dermis (Pelvisoft ${ }^{\circledR}$ Bard, Covington, GA), or bovine (pericardium) [5].

\section{MATERIALS AND METHODS}

Between 2005 and 2007, in the "Santo Bambino" Hospital 74 patients - mean age $64.3 \pm 10.4 \mathrm{SD}$, mean parity $3.2 \pm 1.5 \mathrm{SD}$, and mean BMI 29.8 $\pm 5.3 \mathrm{SD}$ - underwent surgery to repair pelvic organ prolapse (POP). We used the POPQ-System for the clinical evaluation of the prolapse, while the patients were performing a Valsalva manoeuvre in the gynaecological position. Twenty-two patients (29.7\%) had a grade II cystocele, 35 (47.3\%) grade III and 17 (23\%) grade IV. Rectocele was found in 36 women (48.6\%): 15 (41.6\%) had a grade II rectocele, $12(33.4 \%)$ a grade III and $9(25 \%)$ grade IV. The KHQ and PISQ-12 were used to evaluate symptoms and quality of life. During the follow-up the same questionnaires were administered to verify the effectiveness of the operation. The patients' main symptoms were: dyspareunia, urinary retention, palpable mass, difficulty in evacuating. The clinical examination was completed with urodynamic tests. We found stress incontinence (SUI) in 35 cases (47.2\%). The surgical technique adopted in anterior prolapse consists in placing a mesh with a double transobturator approach. On the other hand, in posterior vaginal wall defects we performed high levator myorrhaphy. We used synthetic prostheses - Prolift ${ }^{\circledR}$ (Gynecare, Baltimore,
USA) in 40 cases (54.1\%), Avaulta ${ }^{\circledR}$ (Bard, Covington, GA) in 16 cases (21.6\%) - and biological prostheses Pelvisoft ${ }^{\circledR}$ (Bard, Covington, GA) in the remaining 18 patients (24.3\%). During the same operation we performed colpohysterectomy in 33 cases $(44.5 \%)$, uterosacral ligament fixation in 33 patients (44.5\%) and the TOT technique in 22 cases (29.7\%). The DTOT technique (double transobturator): We pass the mesh through the obturator foramens. It is important to correctly locate the entry points for the four cutaneous incisions in order to create a safe route for the passage of the needles in order to preserve the integrity of the vascular-nervous structures. The first entry points are marked in the genitofemoral fold on the left and right side at the level of the clitoris and the second entry points are located $2 \mathrm{~cm}$ below and $1 \mathrm{~cm}$ lateral from the first ones. Thanks to different studies on cadavers we can observe that the needle, crossing the obturator foramen, stays $3.23 .5 \mathrm{~cm}$ away from the obturator nerve and vessels[6].

\section{RESULTS}

The patients underwent a 3,6, and 12 month follow-up. The double transobturator approach was always safe, there were no intraoperative complications. The mean duration of the operation was 48 minutes for the correction of anterior and posterior prolapse; 117 minutes in the case of associated colpohysterectomy and/or TOT technique. The mean duration of 
hospitalisation was $63 \mathrm{~h}$. The anatomical success rate, defined as a grade of prolapse <2, was 79.7\% (59 patients) for anterior prolapse and 100\% (36 patients) for posterior prolapse. The recurrence rate for anterior prolapse was $20.2 \%$ ( 15 women): 8 patients $(53.3 \%)$ had a grade II prolapse, the remaining $7(46.7 \%)$ a grade III prolapse. Regarding posterior vaginal wall defects we observed complete resolution of the prolapse. Thanks to the questionnaires we calculated that the satisfaction rate of patients was $90.5 \%$ (67 patients). Sixteen patients (41\%) manifested "de novo" incontinence, probably because of the end of the "kinking" effect caused by the cystocele. On the other hand the TOT cure rate was $91.7 \%$. During the follow-up we found vaginal erosions in 5 patients (6.7\%), in 4 cases out of 5 the erosion was located on the anterior colpotomy. Erosion occurred within 6 weeks and 6 months. Two patients were asymptomatic so they were treated with estrogenic topic therapy; on the other hand the remaining three patients had vaginal discharge and dyspareunia, requiring partial excision of the mesh. Seventeen of the $21 \mathrm{pa}$ tients $(80.9 \%)$ with preoperative dyspareunia recovered a normal sexual life.

\section{DISCUSSION}

The double transobturator approach represents an innovation in pelvic floor surgery ; it is based on the concept of anatomical-functional reconstruction using a "tension free" technique. At the beginning of our experience we adopted both synthetic meshes (low density, macroporous and monofilament polypropylene) and biological meshes (porcine derma). Thanks to the analysis of the first data we initially preferred porcine derma because of a lower percentage of erosion than with polypropylene. However, during our follow-up we noticed that women treated with porcine derma had a greater failure rate (recurrence of the prolapse) than those treated with polypropylene, $86.6 \%$ (12 cases) VS $13.4 \%$ (3 cases).

The different behaviour of porcine derma can be explained by its particular structural composition. In fact thanks to its biocompatibility, it stimulates a bland inflammatory reaction and therefore a low risk of erosion, which depends on an excess of this reaction. But, on the other hand, the absence of "cross linking" between its fibres prevents fibroblastic and vascular colonization, so the mesh does not integrate into the host tissue but is encapsulated by it.[7] This involves a lower fixity of the porcine derma to the anatomical structures of the pelvis, so recurrence is more frequent. Because of these considerations the use of porcine derma has been reserved to $\leq$ II grade prolapse. Moreover, synthetic meshes may undergo different complications that can be divided into infective and non-infective complications. Infective complications include functional and anatomical complications.

Functional complications in- 
clude evacuative dysfunction, "de novo" detrusor hyperactivity, stress incontinence, dyspareunia and local chronic pain.

Anatomical complications include foreign body reaction, fibrosis with tissue retraction, calcification, hematoma, thrombosis, erosion, seroma, fistula.

Note that "erosion" is not the same as "infection"; it depends on different risk factors of:

Type of mesh: macroporous or microporous.

Surface of the mesh: Norris et al. were the first to notice that the entity of inflammatory reaction to the mesh is proportional to its surface.

Surgical technique: we have to considerer two important risk factors: excessive tension applied to the mesh, and inadequate dissection of the vaginal wall. To avoid the first risk factor we place the mesh in a "tension free" way. This consists in the placing the mesh under the bladder without any stitches so it can assume the most natural anatomical position possible. Regarding vaginal dissection, if this is too deep it increases the risk of vesical erosion, on the other hand if it is too superficial, it increases the probability of vaginal erosion[8].

Age > 65 years is considered an independent risk factor, which can increase the probability of vaginal erosion 1.6 times. This is caused by the reduction of the tissue's repairing ability due to altered proliferation of the keratinocytes and by changes in the inflammatory response due to alterations of the chemokine content and phagocytic functions. Furthermore in aged skin a reduced synthesis of extracellular matrix is present, which derives from a decrease in metalloproteinase levels. Finally angiogenesis is impaired because of the reduced production of aged microvessels.

Smoking increases the risk of erosion 3 times. Several studies have correlated its influence with wound repair and tissue regeneration. In fact the principal components of tobacco (nicotine, nitric oxide, carbon monoxide) influence wound healing in peripheral tissues; the dermis is particularly sensitive to the vasoconstrictive effects of smoking, and increased levels of carboxyhaemoglobin and of platelet adhesiveness lead to the formation of microthrombi and a reduced ability to deliver oxygen. Finally, increased serum levels of fibrinogen and of haemoglobin, decreased fibrinolytic activity, and direct endothelial injury all contribute to reduce local circulation [9].

Concomitant bysterectomy and anterior inverted T colpotomy: concomitant hysterectomy should be avoided, if necessary an exclusive pericervical colpotomy with retro-dissection of the bladder should be performed, rather than an over extensive $\mathrm{T}$ colpotomy [10]. The main clinical symptoms of erosion are: unspecific pelvic pain, vaginal discharge, urinary and faecal incontinence, dyspareunia. Therapy for erosion includes both medical and surgical treatment; the former consists in topical oestrogen therapy and antibiotic therapy and it is administered to asymptomatic pa- 
tients. On the other hand in symptomatic patients we adopt a surgical approach that consists in partial or total transvaginal excision of the mesh. The choice depends on the type of mesh used: since porcine derma is not integrated into the host tissue, but is only encapsulated, it requires total excision; polypropylene, being totally integrated, only requires partial excision.

At the beginning of our study all the patients with SUI underwent both the operations at the same time. Despite the elevated cure rate (91.7\%), we chose to treat SUI six months later because when we performed the two operations at the same time, there was an increased risk of post-operative bladder retention and overactive bladder symptoms [11]. The choice to treat rectocele with high levator myorrhaphy, without using a mesh, derives from the evidence of a greater incidence of complications connected to the use of prostheses. In fact Huebner et al. describe an unacceptable increase of "de novo" dyspareunia and defaecatory pain [8].

\section{CONCLUSION}

The advent of prostheses in pelvic floor surgery is very important for a definitive resolution of urogenital prolapse. The cure rate, reported in a recent review by Cochrane, ranges from $80 \%$ to $100 \%$ [12]. The use of meshes however, is accompanied by several complications, the most important of which is erosion. Erosion is more frequent when the mesh is introduced vaginally rather than abdominally. Nevertheless the use of the vaginal route is better because the abdominal route involves many complications: increased operative time, mesh infection, bleeding from the retroperitoneal space, wound infection, ureteral injury and small bowel obstruction [13]. The ideal synthetic material should be biocompatible, inert, not carcinogenic, not allergenic, not inflammatory, sterilisable, not modifiable by body tissue, soft and flexible to accommodate the natural stretching of the vaginal tissue during sexual intercourse, and it should be able to avoid shrinkage and mechanical stress, while being easy to handle and readily available at a reasonable cost. At the moment polypropylene mesh represents the first choice in the surgery of urogenital prolapse, because it appears to be associated with a much lower risk of infection and erosion compared with other synthetic grafts ( 0-5\% against $4-30 \%$ of polyethylene, polyester or silicone)[8].Besides, the polypropylene mesh offers a more lasting result than biological prostheses, which were used widely at the beginning, due to the lower rate of erosion, but which today are only used for low grade prolapse because of their high recurrence rate.

In conclusion, if on one hand we have approached the modern idea of the perineum as an integrated functional unit, on the other hand we are still in search of a material that can satisfy standards of permanence of the result without dangerous and sometimes dramatic complications. 


\section{REFERENCES}

1. REBECCA G. ROGERS, D. KAMMERER-DOAK, A. DARROW, K. MURRAY, A. OLSEN, M. BARBER, C. QUALLS :

Sexual function after surgery for stress urinary incontinence and/or pelvic organ prolapse: A multicenter prospective study; in American Journal of Obstetrics and Gynecology.

(2004) 191, 206-10.

2. C. CHALIHA, V. KHULLAR :

Surgical repair of vaginal prolapse: A gynecological hernia; in International Journal of Surgery.

(2006) 4, 242-250.

3. R. DE TAYRAC, G. DEVOLDERE, J. RENAUdie, P. Villard, O. GUilbaud, G. EGLIN :

Prolapse repair by vaginal route using a new protected low-weight polypropylene mesh: 1-year functional and anatomical outcome in a prospective multicentre study; in International Urogynecology Journal. (2007) 18: 251-256.

4. A.M. WEBER, M.D. WALTERS, M.R. PIEDMONTE, L.A. BALLARD : Anterior colporrhaphy: a randomized trial of three surgical techniques; in American Journal of Obstetrics and Gynecology.

(2001) 185(6), 1299-1307.

5. COLIN BIRCH :

The use of prosthetics in pelvic reconstructive surgery; in Best Practice $\&$ Research Clinical Obstetrics and Gynaecology.

(2005) vol. 19, n. 6, pp. 979-991.

6. C. REISENAUER, A. KIRSCHNIAK, U. DREWS, D. WALLWIENER : Anatomical conditions for pelvic floor reconstruction with polypropylene implant and its application for the treatment of vaginal prolapse; in European Journal of Obstetrics \& Gynecology and Reproductive Biology. (2007) 214-225.

7. J.E. ROBLES, J. RIOJA, A. SAIZ, X. BRUGAROLAS, D. ROSELL, J.J. ZUDAIRE, J.M. BERIAN :

Anterior compartment prolapse repair with a bybrid biosynthetic mesh implant technique; in International Urogynecology Journal.

(2007) 18: 1191-1196. 
8. E. MistrangelO, S. MANCUSO, C. NADALINI, D. LIJOI, S. COSTANTINI : Rising use of synthetic mesh in transvaginal pelvic reconstructive surgery; $A$ review of the risk of vaginal erosion; in Journal of Minimally Invasive Gynecology.

(2007) 14, 564-569.

9. F. ARACO, G. GRAVANTE, R. SORGE, D. De VITA, E. PICCIONE : Risk evaluation of smoking and age on the occurrence of postoperative erosions after transvaginal mesh repair for pelvic organ prolapse, in International Urogynecology Journal.

(2008) 19: 473-479.

10. P. COLlinet, F. BELOT, P. DEBODINANCE, E. HA DUC, J.P. LUCOT, M. COSSON :

Transvaginal mesh technique for pelvic organ prolapse repair: mesh exposure management and risk factors; in International Urogynecology Journal. (2006) 17: 315-320.

11. P. HINOUL, H.A.M. BR LMANN, J.P. ROOVERS :

Perspectives in urogynaecology: the surgical challenges abead; in Gynecologica Surgical.

(2008) 5: 3-6.

12. C. MAHER, K. BAESSLER, C.M. GLAZENER et al. :

Surgical management of pelvic organ prolapse in women; in Cochrane Database System Review.

(2004).

13. X. DEFFIEUX, R. DE TAYRAC, C. HuEl, J. BOTTERO, A. GERVAiSE, K. BONNET, R. FRYDMAN, H. FERNANDEZ :

Vaginal mesh erosion after transvaginal repair of cystocele using Gynemesh or Gynemesh-Soft in 138 women: a comparative study; in International Urogynecology Journal.

(2007), 18: 73-79.

For correspondence:

Giuseppe Raciti

via Principe Nicola, 47

95126 Catania,

e-mail: sbuffy@hotmail.it 